\title{
Effectiveness of a Plai Oil Prepared by Thai Traditional Medicine Process in the Treatment of Myofascial Pain Syndrome: A Randomized Placebo Controlled Trial
}

Vanuchawan Wisuitiprot, M.S. ${ }^{1}$, Wasin Bumrungchaichana, B.ATM. ${ }^{2}$, Nipon Kaewtai, M.P.H. ${ }^{2}$,

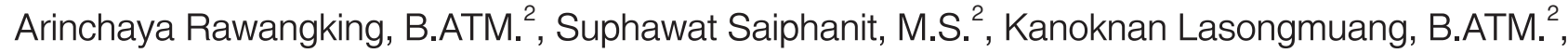
Nitirat Meekai, B.ATM. ${ }^{2}$, Wudtichai Wisuitiprot, Ph.D. ${ }^{1,2,3}$

${ }^{1}$ Cosmetic and Natural Products Research Center, Faculty of Pharmaceutical Sciences, Naresuan University, Mueang, Phitsanulok 65000, Thailand.

2Department of Thai Traditional Medicine, ${ }^{3}$ Herbal Research Unit, Sirindhorn College of Public Health, Wongthong, Phitsanulok 65130, Thailand.

Received 11 February $2019 \bullet$ Revised 26 April $2019 \bullet$ Accepted 1 May $2019 \bullet$ Published online 18 June 2019

\section{Abstract:}

Objective: To evaluate the effectiveness of Plai oil for treating myofascial pain syndrome.

Material and Methods: One hundred and fourteen volunteers with muscle pain from myofascial pain syndrome participated in the study and had Plai oil, placebo oil and diclofenac gel applied to their shoulder and neck for 6 days. Clinical evaluation was determined using visual analogue scales, pressure threshold and cervical range of motion of neck flexion and neck extension measurements.

Results: The results showed that the visual analogue scales of the 3 groups were significantly different from the baseline. The pressure threshold also increased significantly from the baseline $(3.87 \pm 1.36)$ in the volunteers who applied Plai

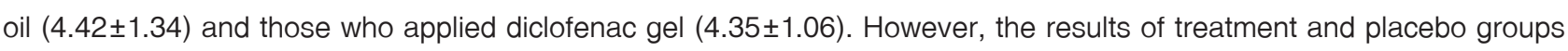
at the last follow-up were not significantly different. Interestingly, it was observed that Plai oil and placebo oil significantly increased the angle of neck flexion and extension within 3 days of application. Muscle pain treatment with Plai oil resulted in a good outcome that was no different to the outcome of applying the diclofenac gel and placebo.

Contact: Wudtichai Wisuitiprot, Ph.D.

${ }^{1}$ Cosmetic and Natural Products Research Center, Faculty of Pharmaceutical Sciences, Naresuan University, Muaeng, Phitsanulok 65000, Thailand.

${ }^{2}$ Department of Thai Traditional Medicine, ${ }^{3} \mathrm{Herbal}$ Research Unit, Sirindhorn College of Public Health, Wongthong, Phitsanulok 65130, Thailand.

E-mail: wudtichai@scphpl.ac.th
J Health Sci Med Res 2019;37(3):207-215 doi: 10.31584/jhsmr.201954 www.jhsmr.org 
Conclusion: It was demonstrated that Plai oil is as effective for relieving myofascial pain as $1.0 \%$ diclofenac gel. The interpreted results of muscle pain are not fully clarified due to placebo effect and other influencing parameters. However, Plai oil also decreased muscle tension and improved the restricted range of motion. We can recommend that Plai oil can be used as an alternative topical application for muscle pain treatment.

Keywords: myofascial pain, Plai oil, Zingiber montanum

\section{Introduction}

Zingiber montanum (Z. montanum) (J.Koenig) Link ex A.Dietr. is a traditional herb that has been used for treating muscle pain and inflammation. In Thailand, Z. montanum also known as Plai, is used in traditional Thai medicine and has several applications. ${ }^{1,2}$ Plai is the main ingredient in Thai herbal compresses that are used for treating muscle pain, particularly in combination with Thai massage. ${ }^{3}$ Many reports have revealed that Plai is composed of several compounds that demonstrate anti-inflammatory activity. These bio-active compounds include curcuminoids, terpenoids and phenylbutaniods which are prominent in the essential oils and crude extracts from Plai rhizomes. ${ }^{4-6}$ Phenylbutaniods are important components that possess anti-inflammatory properties, particularly (E)-4 (3',4'-dimethyl-phenyl) but-3-en-1-ol (compound D), and (E)-1-(3,4-dimethoxyphenyl) butadiene (DMPBD). ${ }^{4}$ Previous reports indicated that phenylbutaniod derivatives from Plai rhizomes show anti-inflammatory activity with mechanisms similar to non-steroidal antiinflammatory drugs (NSAIDs). ${ }^{4,7}$ DMPBD and compound $D$ have been reported as being effective in mice in treatments involving the inhibition of inflammation induced by carrageenan and diethyl phenylpropiolate. ${ }^{8,9}$ The results from both in vitro and in vivo studies have suggested that the possible mechanisms were the inhibition of prostaglandins synthesis and the decrease of inflammatory mediator migration. Considering the potential of antiinflammatory activity, researchers have attempted to prove the effectiveness of Plai in clinical studies. Plygesal cream containing $14.0 \%$ Plai essential oil showed an obvious effectiveness in ankle inflammation treatment ${ }^{10}$, a result that correlates well with a recent clinical study that reported the effectiveness of an emulsion containing $14.0 \%$ essential Plai oil for decreasing muscle soreness after exercise. ${ }^{11}$

For a long time, Thai traditional medicine has used Plai oil for treating muscle inflammation and arthritis. Doctors of Thai traditional medicine have prepared Plai oil by frying the fresh rhizomes of $Z$. montanum ${ }^{12}$ together with plant fixed oil. Plai oil, a yellowish oil, is usually used for muscle pain treatment in combination with Thai massage. Plai oil has been listed in the National List of Essential Medicine published by the Ministry of Public Health, Thailand which recommends Plai oil for treating muscle and joint inflammation. Although the Plai oil preparation method has been suggested for a long time, the medicinal effectiveness of Plai oil has never been clinically evaluated. Hence, the objective of this study was to evaluate the safety and effectiveness of Plai oil prepared using the Thai traditional method. The effectiveness of the formulations as a muscle pain treatment was evaluated using visual analogue scales, an algometer, and an inclinometer.

\section{Material and Methods}

\section{Materials}

Rhizomes of Z. montanum (J.Koenig) Link ex A.Dietr. with an age of 1 year were purchased from Khaokho herbary 
organic farm, Phetchaboon province, Thailand. Organic solvents used in this study were analytical reagent grade, purchased from Labscan (V.S. Chemhouse, Thailand). Palm oil (Oleen ${ }^{\circledR}$, Thailand) was purchased from the market in Phitsanulok province.

\section{Plai oil preparation}

The fresh rhizomes of $Z$. montanum used in the study were chopped into tiny pieces and then fried with palm oil until they were brownish and crunchy. The frying time was approximately 1 hour. The yellowish oil was purified by paper filtration, and the filtered Plai oil was kept in a cool place and protected from light. The ratio of fresh rhizomes and palm oil was $1: 3$ weight by weight.

\section{Oil formulations}

The oil formulations that were used in the clinical study were prepared in 2 formulations, with one to be used as a placebo; the ingredients of each are shown in Table 1.

Table 1 The ingredients of oil formulation in study

\begin{tabular}{lll}
\hline \multirow{2}{*}{ Ingredients } & \multicolumn{2}{c}{ Content (\%) } \\
\cline { 2 - 3 } & Plai oil & Placebo oil \\
\hline Fried Plai oil & 91.5 & - \\
Methyl salicylate & 5.0 & 5.0 \\
Eucalyptus oil & 3.0 & 3.0 \\
Butylhydroxytoluene & 0.5 & 0.5 \\
Palm oil & - & 91.5 \\
\hline
\end{tabular}

\section{Clinical study process}

This study was performed at the Tikitchavej Thai Traditional Medicine Clinic in the Department of Thai Traditional Medicine, Sirindhorn College of Public Health, Phitsanulok, Thailand. The study was approved by the Ethic
Committee for Research in Human Subjects in The Field of Thai Traditional and Alternative Medicine, Department of Thai Traditional and Alternative Medicine, Ministry of Public Health, Thailand with reference number 12/2559. The research protocol was registered and approved by the Thai Clinical Trial Registry: registered number TCTR20190127002.

One hundred and fourteen volunteers aged between 18-60 years participated. All participants gave their written informed consent. The inclusion criteria were simply that the volunteer had been diagnosed with myofascial pain by a doctor of Thai traditional medicine. The trigger points of pain were localized at the neck and shoulder areas. The exclusion criteria: the volunteers could not be pregnant or currently using anti-inflammatory drugs. The volunteers were allocated into 3 groups by block randomization (38 volunteers per group): group 1, Plai oil; group 2, placebo oil; and group 3, 1.0\% diclofenac gel. For all the groups, a dose of 4 grams of the oils or gel was gently rubbed into the neck and shoulder areas for about 5 minutes until the oils or gel were not visible. The volunteers were similarly instructed to apply the oils or gel twice a day for 6 days (morning and evening). During the study period, volunteers were evaluated on day 3 and day 6 . The remaining oil or gel was recorded along with their weight at every follow-up for monitoring volunteer compliance.

\section{Clinical evaluation}

All of the volunteers were evaluated for muscle pain using a visual analogue scale in the range from 0 to 10 (0 indicating no pain, 10 indicating worst pain). The trigger points were found in the pain area and the pain severity was measured using an algometer (Wagner Instruments, Greenwich, USA); the pressure threshold measurement of the trigger point represented the muscle pain. Muscle tension was assessed by measuring the cervical range of motion of the neck, with the angles of cervical motion determined in 2 directions; neck flexion 
and neck extension, to access the volunteer's muscle tension, and were measured using an inclinometer. All parameters were evaluated at the starting time, and then were compared at each follow-up, with the exception of the visual analogue scales where the starting time values were compared at the end of the study. Skin irritation was also observed during the study period. Paired sample t-test was used to compare the baseline and post-treatment changes in outcome variables in each group. Analysis of covariance was used to detect any statistically significant differences in the improvement between the three groups.

\section{Results}

There were 114 volunteers enrolled in this study. The general characteristics of volunteers who were recruited in the study are presented in Table 2. The study process was performed according to Figure 1. No volunteer had skin irritation during the study period.

\section{Visual analogue scale}

Muscle pain was subjectively identified using a visual analogue scale. At the starting time, the muscle pain scores were not significantly different among the 3 groups at the baseline. Table 3 shows that both of the oil formulations stated in Table 1, and the diclofenac gel, showed a significant decrease in visual analogue scale
(VAS) score from the baseline, with no significant difference in the decrease of muscle pain reported between the two oil formulations and the gel $(p-$ value $<0.05)$.

Table 2 Demographic data of recruited volunteers

\begin{tabular}{ll}
\hline Index & Data \\
\hline Age ranges (years) & $18-50$ \\
Average age (years \pm S.D.) & $32.92 \pm 12.15$ \\
Sex & \\
$\quad$ Male & $11(9.6 \%)$ \\
$\quad$ Female & $103(90.4 \%)$ \\
VAS score (mean \pm S.D.) & $5.25 \pm 1.23$ \\
Pressure threshold measurement (mean $\pm S . D)$. & $4.56 \pm 0.56$ \\
Cervical range of motion & \\
$\quad$ Neck flexion (mean $\pm S . D)$. & $54.28 \pm 2.53$ \\
$\quad$ Neck extension (mean $\pm S . D)$. & $50.44 \pm 0.72$ \\
\hline
\end{tabular}

S.D.=standard deviation

\section{The pressure threshold measurement}

The trigger points presenting pain severity were measured using an algometer that showed the value. The severity of muscle pain showed an inverse relationship with the pressure threshold force from the algometer. ${ }^{13,14}$ Muscle pain severity as assessed by the pressure force from the algometer of each treatment group is presented in Figure 2. The results indicate that Plai oil significantly increased the pressure threshold at the second followup (T1) when compared to the baseline (T0) while the

Table 3 Volunteer pain evaluation comparing before to after treatment using visual analogue scales

\begin{tabular}{|c|c|c|c|}
\hline \multirow{2}{*}{ Sample groups } & \multicolumn{3}{|c|}{ VAS score (mean \pm S.D.) } \\
\hline & Before treatment & After treatment & Change of score \\
\hline Placebo oil (n=38) & $5.71 \pm 2.17$ & $3.87 \pm 2.19^{*}$ & $1.93 \pm 1.36$ \\
\hline Diclofenac gel $(n=38)$ & $5.66 \pm 1.47$ & $4.36 \pm 1.86^{*}$ & $1.67 \pm 1.23$ \\
\hline Plai oil $(n=38)$ & $5.94 \pm 2.10$ & $3.81 \pm 1.69^{*}$ & $2.26 \pm 1.44$ \\
\hline
\end{tabular}

*significantly different from before treatment ( $p$-value<0.05)

VAS=visual analogue scale, S.D.=standard deviation 
placebo oil did not increase that value until the last followup, on day 6. The volunteers who applied the diclofenac gel showed a significant increase $(p-v a l u e<0.05)$ in pressure threshold values at the end of the study. Due to significantly different of pressure threshold at baseline, the correlation of pressure threshold analyzed by analysis of covariance at the last follow-up indicated that no significant difference of pressure threshold between 3 groups on day 6 .

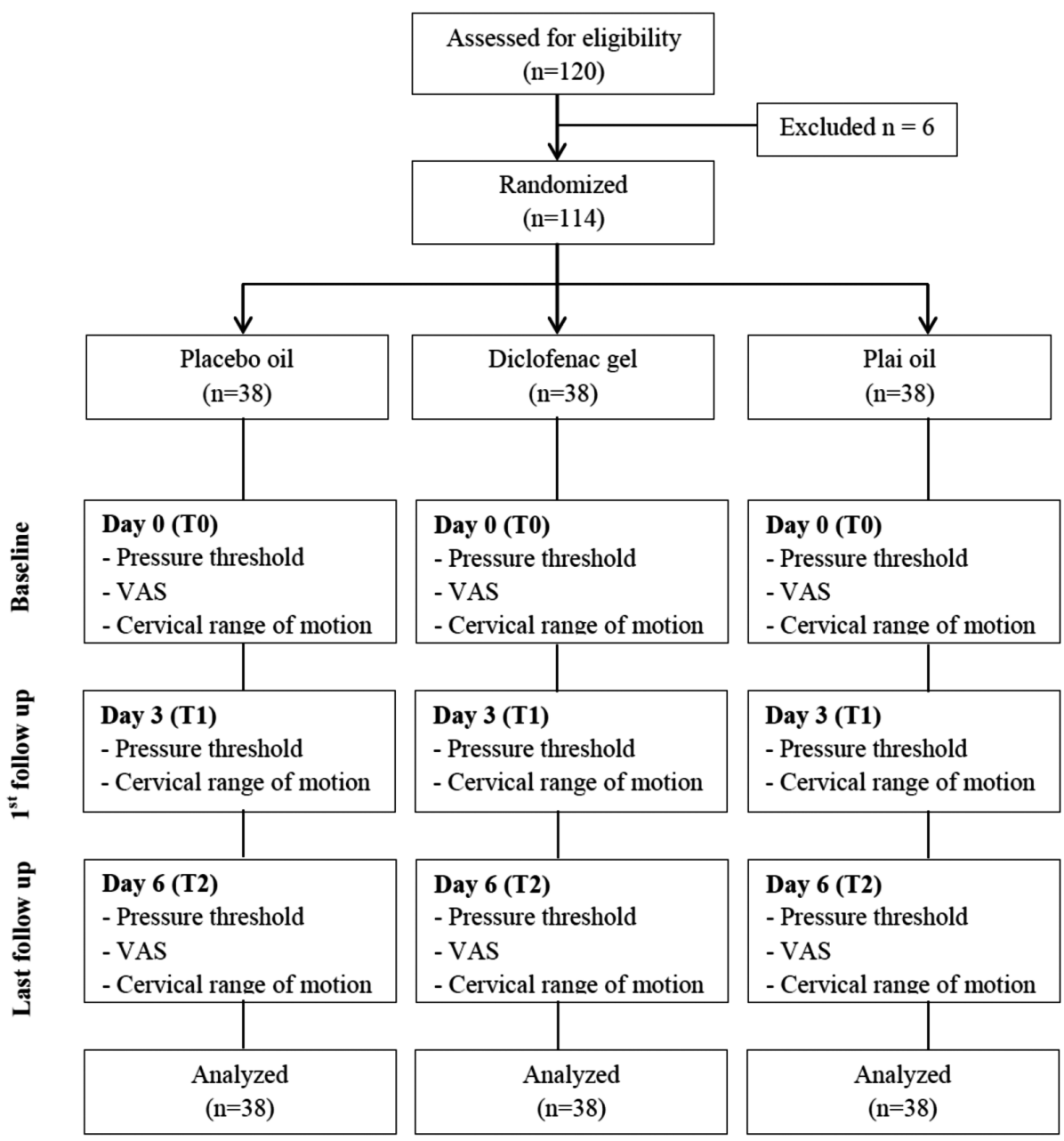

VAS=visual analogue scale, T0=baseline, $\mathrm{T} 1=$ first follow-up, $\mathrm{T} 2=$ =second follow $-\mathrm{up}$

Figure 1 Flow diagram and summary outcome of study 


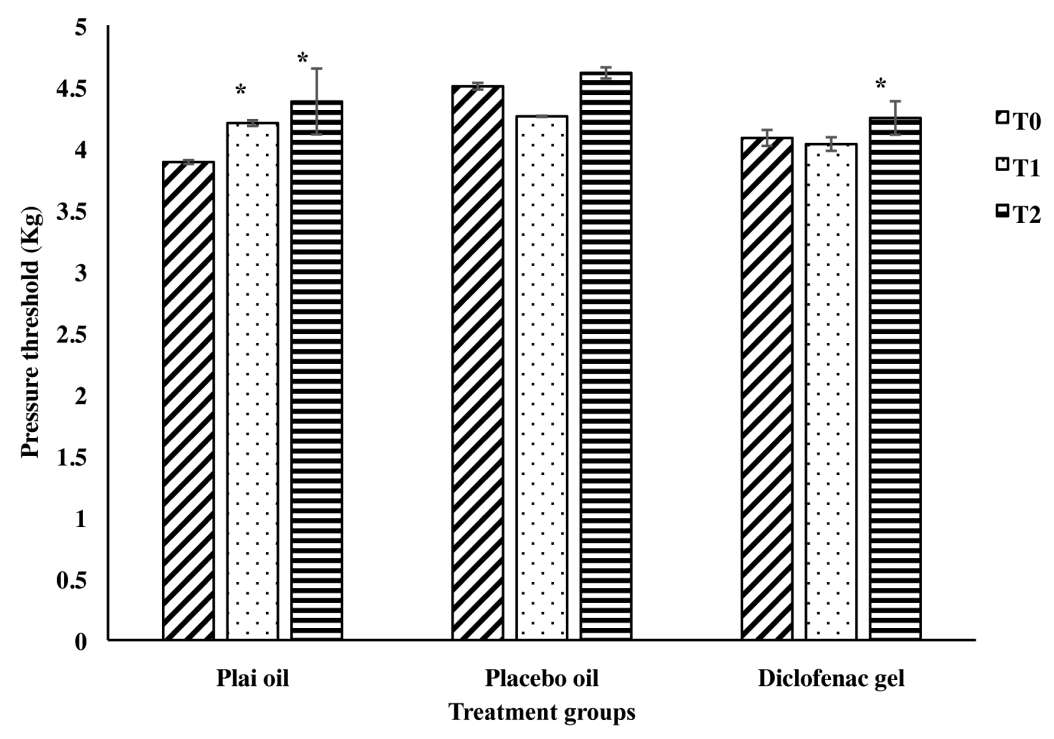

${ }^{*} \mathrm{p}$-value $<0.05, \mathrm{~T} 0=$ baseline, $\mathrm{T} 1=$ first follow-up, $\mathrm{T} 2=$ second follow-up

Figure 2 The pressure threshold of trigger points measured from each group comparing among baseline, first followup and the last follow-up

Table 4 The cervical range angle accessed by neck flexion and extension in each group compared with follow-up times

\begin{tabular}{|c|c|c|c|c|c|c|}
\hline \multirow{3}{*}{ Sample groups } & \multicolumn{6}{|c|}{ Cervical range of motion (neck flexion and neck extension) } \\
\hline & \multicolumn{2}{|c|}{ T0 } & \multicolumn{2}{|c|}{ T1 } & \multicolumn{2}{|c|}{ T2 } \\
\hline & NF & NE & NF & NE & NF & NE \\
\hline Placebo oil $(n=38)$ & $51.83 \pm 12.15$ & $50.04 \pm 13.48$ & $56.67 \pm 10.57^{*}$ & $54.26 \pm 11.34^{*}$ & $57.57 \pm 11.90^{*}$ & $53.74 \pm 10.18^{*}$ \\
\hline Diclofenac gel $(n=38)$ & $56.90 \pm 14.07$ & $51.29 \pm 11.44$ & $60.45 \pm 14.36$ & $54.46 \pm 13.40$ & $59.16 \pm 12.93$ & $53.36 \pm 11.06$ \\
\hline Plai oil $(n=38)$ & $54.12 \pm 12.72$ & $50.01 \pm 12.69$ & $59.44 \pm 11.47^{*}$ & $56.63 \pm 63^{*}$ & $60.04 \pm 13.11^{\star \star}$ & $57.19 \pm 12.74^{*}$ \\
\hline
\end{tabular}

$\mathrm{NF}=$ neck flexion, $\mathrm{NE}=$ neck extension, $\mathrm{T0}=$ baseline, $\mathrm{T} 1=$ first follow-up, $\mathrm{T} 2=$ second follow-up, ${ }^{*} \mathrm{p}-\mathrm{value}<0.05$

\section{Cervical range of motion measurement}

An inclinometer was used for determining the cervical range of motion. The cervical angle of each volunteer was measured at each follow-up. Neck flexion and extension values are presented in Table 4. The table shows that the volunteers who applied Plai oil achieved a significant increase in the angles in both neck flexion and extension over the baseline at the day 3 follow-up. The results from the placebo oil group were also significantly different in both directions from baseline. The increase of cervical angle of volunteer applying placebo or Plai oils still shows significantly different from baseline until day 6 follow-up. Interestingly, the significant difference of cervical range angle between baseline and treatment of volunteer achieved the placebo oil or Plai oil was not evident in diclofenac gel group. 


\section{Discussion}

The current study revealed that Plai oil that was prepared by frying the rhizomes with plant fixed oil showed significant effectiveness for relieving muscle pain in the volunteers who suffered myofascial pain syndrome. The muscle pain evaluation of the volunteers was that all 3 formulations were significantly effective as a muscle pain treatment. Result of VAS score indicated that all of the volunteers evaluates the decrease of muscle pain after applying the formulations for 6 days. Although Plai oil and diclofenac gel showed the effectiveness in muscle pain treatment, placebo oil also decreased muscle pain when comparing with baseline. Normally, the decrease in muscle pain as a result of the inhibition of cyclooxygenase (COX) enzymes, prostaglandins synthesis is decreased and the inflammation process is then limited. Plai oil is composed of (E)-1-(3,4-dimethoxyphenyl) DMPBD, (E)1-(3,4-dimethoxyphenyl) compound D and curcumin those present strong inhibitors of COX.,8,15 It is well known that those compounds have an anti-inflammatory effect of reducing the activities of COX-2, lipoxygenase, inducible nitric oxide synthase and inflammatory cytokines. ${ }^{15}$ Previous clinical studies of topical formulations of Plai cream performed by essential oil of $Z$. montanum confirm that volunteers reported the effectiveness of Plai applications as a muscle pain treatment. ${ }^{11,16}$ Diclofenac sodium is the NSAIDs that relieves muscle pain by inhibiting the activity of COX influencing prostaglandins synthesis in the arachidonic acid metabolism pathway. In the case of the placebo oil, the volunteers also evaluated the placebo oil as giving significant relief to muscle pain when compared to the baseline. It is most likely that $5.0 \%$ methyl salicylate incorporated in the placebo oil decreases the muscle inflammation by inhibiting the synthesis of proinflammatory cytokines, such as interluekin-1 beta and tumor necrosis factor-alpha; moreover, methyl salicylate can increase local blood circulation by a counterirritant property; which has been attributed to the activation and desensitization of the cutaneous nerve. Thus the muscle pain is reduced ${ }^{17,18}$, and the warm feeling experienced and skin desensitization which occurs when applying methyl salicylate and aroma oil to the skin could help the volunteer with pain relief. However, the Food and Drug Administration of the United States of America recommends that $10.0 \%$ methyl salicylate incorporated into the topical patch can give the effectiveness in muscle pain treatment when covering the inflammation site for 1 hour $^{17}$, and thus so the application of $5.0 \%$ methyl salicylate was not enough to alter the trigger point within the short time period. Hence, the pressure threshold of the trigger point of volunteers in the placebo group was not different from the baseline. In addition, the pressure threshold determined by an algometer indicated that both the Plai oil formulations and the diclofenac gel increased the pressure threshold after application, with the Plai oil significantly increasing the pressure threshold within 3 days. The increase in the pressure threshold implies an improvement in the level of muscle pain. ${ }^{13,19}$ Our current study showed the effectiveness of diclofenac gel as a muscle pain treatment to be significant after 6 days of the treatment program. A few studies have indicated that the chemical components of Z. montanum, particularly compound $D$, presented antiinflammatory activity similar to diclofenac. ${ }^{7}$ According to VAS score, Plai oil, placebo oil and diclofenac gel may decrease muscle pain effectively with mechanism mentioned first, however the pressure threshold of volunteer from placebo groups at the last follow-up was not significantly different when comparing to baseline. On the contrary, the pressure threshold at the last follow-up of each group also was not significantly different. The discrepancy in the results might be due to the efficacy of 3 the formulations for altering the trigger point being no different. Being incorporated, the Plai oil in the formulations did not show a more advantageous effect on muscle pain than the placebo oil. 
Although the active compound of $Z$. montanum is identified as an anti-inflammatory agent, the chemical components and their stability, particularly compound D, of Plai oil applied by the traditional process have not been fully identified. There was a finding that compound $D$ was more degraded when exposed to high temperatures. ${ }^{20}$ The efficacy of both oil formulations may be caused by methyl salicylate.

The cervical range of motion can be used to identify muscle tension. A patient who has myofascial pain usually presents with limited cervical range angles due to muscle tension and inflammation. ${ }^{21}$ Neck flexion and extension were significantly increased from the baseline with both the Plai oil and the placebo oil. Both oil formulations showed effectiveness for muscle relaxation that was accessed by increasing the angles of neck flexion and extension while the diclofenac gel did not show a significant difference from the baseline. The discrepancy in the results may be caused by the method of applying the oils and gel by the volunteers in the 3 groups. According to volunteer's comment, after applying the oil formulation, the volunteers often self-massage at the application site in order to enhance the absorption, whereas the gel formulation is quite dry and does not require the self-massaging process. As massage is an effective method for decreasing muscle tension ${ }^{22,23}$, the self-massage activity of the oil formulation volunteers may well have had an effect. ${ }^{24}$

\section{Conclusion}

Plai oil was demonstrated as being effective for relieving myofascial pain. It reduced the trigger point formation within 3 days after the first application. Our results indicated that the application of Plai oil significantly decreased the muscle pain in the volunteers from the baseline at both the 3 days follow-up and after 6 days. Plai oil was also shown to diminish muscle tension by increasing the ability for muscle stretching. Muscle pain treatment with Plai oil was most satisfactory, as was the diclofenac gel, except that the diclofenac gel did not have the effect of decreasing muscle tension. However, the effectiveness of Plai oil is not fully clarified due to the placebo effect, muscle pain relief may be cause from other ingredients in formula particularly methyl salicylate. The active components of Plai oil has to be further studied for clarifying the effectiveness of Plai oil in muscle pain treatment.

\section{Acknowledgement}

The study was supported with a grant from the Thai Traditional Medical Knowledge Fund, Department of Thai Traditional and Alternative Medicines, the Ministry of Public Health, Thailand.

\section{Conflict of interest}

The authors have no conflict of interest to declare.

\section{References}

1. Picheinsunthorn C, Shavarit M, Jirawong W. Description of Tumra Osoth Pranarai. Bangkok: Amarin; 2008.

2. Hanpithakpong W. Studies on safety and efficacy of Phlai gel. Bangkok: Graduate School, Mahidol University; 1999.

3. Suksaeree J, Charoenchai L, Pichayakorn W, Boonme P. HPLC method development and validation of (E)-4-(3,4-dimethoxyphenyl)-but-3-en-1-ol in Zingiber cassumunar Roxb. from Thai herbal compress ball. Int J Pharm Pharm Sci 2013;3:115-7.

4. Lateh L. Development of extraction method for phenylbutanoids from Zingiber cassumunar Roxb. Songkhla: Faculty of Pharmaceutical Sciences, Prince of Songkla University; 2013.

5. Department of Medical Sciences MoPH. Thai herbal pharmacopoeia. Vol 1. Bangkok: Prachachon; 2007.

6. Suksaeree J, Monton C, Charoenchai L, Madaka F, Chusut T. Determination of (E)-4-(3',4'-Dimethoxyphenyl)-But-3en-1-ol Content in Zingiber cassumunar Roxb. (Plai) Patches. Int J Pharm Pharm Sci 2014;6:434-6.

7. Han AR, Kim MS, Jeong YH, Lee SK, Soe EK. Cyclooxygenase-2 inhibitory phenylbutenoids from the rhizomes of Zingiber cassumunar. Chem Pharm Bull 2005;53:1466-8.

8. Jeenapongsa R, Yoovathaworn K, Sriwatanakul KM, 
Pongprayoon U, Sriwatanakul K. Anti-inflammatory activity of (E)-1-(3,4-dimethoxyphenyl) butadiene from Zingiber cassumunar Roxb. J Ethnopharmacol 2003;87:143-8.

9. Panthong A, Kanjanapothi D, Niwatananant W, Tuntiwachwuttikul $P$, Reutrakul V. Anti-inflammatory activity of compound $D$ $\left\{(E)-4-\left(3^{\prime}, 4^{\prime}\right.\right.$-dimethoxyphenyl)but-3-en-2-ol $\}$ isolated from Zingiber cassumunar Roxb. Phytomedicine 1997;4:207-12.

10. Loupattarakasem W, Kowsuwon W, Laupattarakasem P, Eungpinitpong W. Efficacy of Zingiber cassumunar ROXB. (Plygesal) in the treatment of ankle sprain. Srinagarind Med J 1993;8:159-64.

11. Manimmanakorn N, Manimmanakorn A, Boobphachart D, Thuwakum W, Laupattarakasem W, Hamlin MJ. Effects of Zingiber cassumunar (Plai cream) in the treatment of delayed onset muscle soreness. J Integr Med 2016;14:114-20.

12. Health MoP. National list of essential medicine 2016. In: Drugs DoP, editor. National list of essential medicine 2016. Nonthaburi: Ministry of Public Health; 2016;p.269.

13. Park G, Kim CW, Park SB, Kim MJ, Jang SH. Reliability and usefulness of the pressure pain threshold measurement in patients with myofascial pain. Ann Rehabil Med 2011;35: 412-7.

14. Manimmanakorn N, Manimmanakorn A, Boukamtuem T. Reliability and validity of simple algometer. J Thai Rehabil Med 2005;15:79-87.

15. Srimal RC, Dhawan BN. Pharmacology of diferuloyl methane (curcumin), a non-steroidal anti-inflammatory agent. J Pharm Pharmacol 1973;25:447-52.

16. Pongprayoon $U$, Soontornsaratune $P$, Jarikasem $S$, Sematong $T$, Wasuwat S, Claeson P. Topical antiinflammatory activity of the major lipophilic constituents of the rhizome of Zingiber cassumunar. Part I: the essential oil. Phytomedicine 1997;3: $319-22$.
17. Higashi Y, Kiuchi T, Furuta K. Efficacy and safety profile of a topical methyl salicylate and menthol patch in adult patients with mild to moderate muscle strain: a randomized, doubleblind, parallel-group, placebo-controlled, multicenter study. Clin Ther 2010;32:34-43.

18. Zhang D, Liu R, Sun L, Huang C, Wang C, Zhang DM, et al. Anti-inflammatory activity of methyl salicylate glycosides isolated from Gaultheria yunnanensis (Franch.) Rehder. Molecules 2011;16:3875-84.

19. Fischer AA. Pressure threshold meter: its use for quantification of tender spots. Arch Phys Med Rehabil 1986;67:836-8.

20. Janpim K, Nualkaew S, Priprem A. Stability testing of a Plai extract. Paper presented at: Graduate Research Conference; 2012 Feb 16-17; Khonkaen. Khonkaen: Graduated School, Khonkaen University; 2012.

21. Finley JE. Physical medicine and rehabilitation for myofascial pain [homepage on the Internet]. New York: Medscape [cited 2015 Aug 12]. Available from: http://emedicine.medscape. com/article/313007-overview

22. Boonruab J, Niempoog S, Pattaraarchachai J, Palanuvej C, Ruangrungs $\mathrm{N}$. Effectiveness of the court-type traditional Thai massage versus topical diclofenac in treating patients with myofascial pain syndrome in the upper trapezius. Indian J Traditional Knowledge 2016;15:30-4.

23. Kong LJ, Zhan HS, Cheng YW, Yuan WA, Chen B, Fang M. Massage therapy for neck and shoulder pain: a systematic review and meta-analysis. Evid Based Complement Alternat Med 2013. doi: 10.1155/2013/613279.

24. Bervoets DC, Luijsterburg PAJ, Alessie JJN, Buijs MJ, Verhagen AP. Massage therapy has short-term benefits for people with common musculoskeletal disorders compared to no treatment: a systematic review. J Physiother 2015;61:106-16. 